\title{
Producción Científica sobre Narrativa en Educación Matemática en la Web of Science
}

\section{Scientific Production about Narrative in Mathematics Education field in the Web of Science}

\author{
Rafael Bracho* \\ Noelia Jiménez-Fanjul ${ }^{* *}$ \\ Alexander Maz-Machado ${ }^{* * *}$ \\ Manuel Torralbo-Rodríguez ${ }^{* * * *}$ \\ Antonio Fernández-Cano ${ }^{* * * * *}$
}

\begin{abstract}
Resumen
En este trabajo se realiza un estudio cienciométrico de la producción investigadora sobre narrativa en educación matemática indexada en las bases de datos de la Web of Science de Thomson Reuters. Se ha analizado diacrónicamente, se han estudiado diversos indicadores de producción y de citación desde las perspectivas de autoría, vinculación institucional y nacionalidad de los autores. Así mismo, se ha realizado un estudio de redes sociales aplicado a la autoría y colaboración institucional. También se analizó la relación temática a partir de la cocitación de las palabras-clave que caracterizan los trabajos. Se ha constatado que la investigación en narrativa en educación matemática está empezando a adquirir una presencia relativamente consolidada en los últimos años, si bien aún no puede hablarse de grandes autores ni de colegios invisibles especializados. En el análisis
\end{abstract}

\footnotetext{
* Doctor en Matemáticas por la Universidad de Córdoba (UCO). Profesor de Didáctica de la Matemática. Departamento de Matemáticas, Universidad de Córdoba (UCO), Córdoba, España. Dirección postal: Facultad de Educación, Avda San Alberto Magno, s/n, 14071, Córdoba, España. E-mail: ma1brlpr@uco.es.

** Máster en Educación por la Universidad de Córdoba (UCO). Profesora de Didáctica de la Matemática. Departamento de Matemáticas, Universidad de Córdoba (UCO), Córdoba, España. Dirección postal: Facultad de Ciencias de la Educación, Avda San Alberto Magno, s/n, 14071, Córdoba, España. E-mail: noelia.jimenez@uco.es.

*** Doctor en Matemáticas por la Universidad de Granada (UGR). Profesor de Didáctica de la Matemática. Departamento de Matemáticas, Universidad de Córdoba (UCO), Córdoba, España. Dirección postal: Facultad de Ciencias de la Educación, Avda San Alberto Magno, s/n, 14071, Córdoba, España, España. E-mail: ma1mamaa@uco.es.

**** Doctor en Matemáticas por la Universidad de Granada (UGR). Profesor de Didáctica de la Matemática. Departamento de Matemáticas, Universidad de Córdoba (UCO), Córdoba, España. Dirección postal: Facultad de Ciencias de la Educación, Avda San Alberto Magno, s/n, 14071, Córdoba, España, España. E-mail: ma1torom@uco.es.

***** Doctor en Filosofía y Ciencias de la Educación (Pedagogía) por la Universidad de Granada (UGR). Catedrático de Métodos de Investigación y Diagnóstico en Educación. Departamento de Métodos de Investigación y Diagnóstico en Educación, Universidad de Granada (UGR), Granada, España. Dirección postal: Facultad de Educación, Campus de Cartuja, 18071, Granada, España. E-mail: afcano@ugr.es.
} 
temático realizado se halló un vínculo entre los descriptores de narrativa en educación matemática con aspectos relacionados con el perfil del profesorado de matemáticas.

Palabras-clave: Educación Matemática. Narrativa. Indicadores bibliométricos. Web of Science. Investigación.

\begin{abstract}
In this paper we present a scientometric study of narrative production in mathematics education field indexed in the Web of Science databases. We analyze this time series diachronically as well as we study several indicators of scientific production and citation from different perspectives, such as authorship, institutional affiliation, and nationality of the authors. An analysis of social networks applied to authorship and institutional collaboration between countries has been carried out. Moreover we study the thematic relationship established by the author's keywords that characterize the production. It was found that the narrative research in mathematics education is starting to acquire a relatively consolidated presence in recent times, but we cannot still speak of great producers or specialized invisible colleges. The thematic analysis shows a link between narrative descriptors in mathematics education with others related to aspects of the mathematics teacher profile.
\end{abstract}

Keywords: Mathematics Education. Narrative. Bibliometric indicators. Web of Science. Research.

\title{
1 Introducción
}

En el año 2011 se realizó el I Congreso Iberoamericano de la Historia de la Educación Matemática (CIHEM) en Covilha, Portugal. Allí se hizo notorio el gran número de trabajos presentados que tenían como hilo conductor las historias de vida asociadas a la formación del profesorado y a la historia de la educación matemática (en adelante EMA) y en ellas la narrativa era el método empleado para desarrollar las investigaciones. Un amplio sector de investigadores sacaron a la luz la narrativa en educación matemática (en adelante narrativa EMA) como una metodología que se venía desarrollando con fuerza en algunos de los países iberoamericanos, especialmente en Brasil. Ejemplo de esto son los trabajos de Cury (2010) y Miskulin et al. (2012), que abarcan tanto el uso de la narrativa para el análisis de la historia de la educación matemática como una reflexión docente para la mejora del profesorado a través del uso de narrativas.

Esta irrupción de la narrativa EMA es fácil de entender si tenemos en cuenta que la educación matemática es una disciplina emergente que recibe aportaciones desde diversas ramas del conocimiento tales como la psicología, la pedagogía, la sociología, las matemáticas, la historia de las matemáticas etc. (STEINER, 1990; GODINO, 1991). Por tal razón, la investigación que se realiza en ella aglutina una gran variedad tanto de tópicos y objetivos de interés como de estrategias metodológicas.

Sin embargo, la educación matemática como disciplina científica presenta una serie de características particulares que la distinguen de otras disciplinas, y esto es algo que se constata, evidentemente, en los trabajos de investigación. Esta especificidad no permite tomar 
simplemente prestadas las técnicas de investigación, metodologías y teorías de las disciplinas vecinas. Como afirman Sierpinska y Kilpatrick (1998), normalmente se requiere una adaptación o teorías originales y las metodologías tienen que tener un desarrollo desde el propio ámbito de la educación matemática.

Desde diversos planteamientos se ha buscado identificar tanto los objetivos de la investigación en educación matemática como los métodos para realizarla. Así, Johnson (1980a; 1980b) basó la metodología en cinco categorías de investigación: encuesta, experimento, estudio de caso, evaluación y filosófico/histórico, y narrativa EMA encaja de forma adecuada dentro de las tres últimas categorías.

Por otro lado, Ernest (1998, p. 75) señala como uno de los objetos de interés de la investigación en EMA "las instituciones sociales de la educación matemática: las personas, lugares, instituciones (universidades, colegios, centros de investigación), congresos, organizaciones, redes, revistas etc. y su relación con otros contextos sociales" y, sin duda, son precisamente las personas las protagonistas de las narrativas y las historias de vida de muchas de las investigaciones en EMA, especialmente en historia de la educación matemática.

Se han realizado estudios para conocer de forma general cuáles son los métodos de investigación utilizados en EMA reflejados en los artículos científicos (DONOGHUE, 1999), en congresos (GODINO et al., 2012) y en tesis doctorales (TORRALBO et al., 2004), pero no se conocen trabajos que indaguen sobre qué se ha publicado relacionado con una metodología o temática especifica en EMA.

En algunas disciplinas existe la tradición de realizar periódicamente estudios cienciométricos sobre la producción científica (BEHRENS; LUKSH, 2011; GARCÍAMARTÍNEZ; GUERRERO-BOTE; MOYA-ANEGÓN, 2012), lo que permite tener una visión global de ellas y detectar tendencias, patrones y regularidades que muestran hacia dónde está evolucionado la disciplina o cuáles son los temas o tópicos que generan interés por la demanda social o por el impulso de determinados colectivos, sean estos económicos, políticos o sociales. La educación matemática no es ajena a estas influencias, y por tanto, es necesario realizar estudios de esta naturaleza. Por ello, y ante el creciente interés por la narrativa en EMA, consideramos pertinente y de actualidad realizar un análisis de la producción científica relacionada con la narrativa EMA y publicada en revistas indexadas en las bases de datos de la Web of Science (WoS). Para ello, a grandes rasgos, nos proponemos los siguientes objetivos:

1. Realizar un análisis cienciométrico de la producción en narrativa EMA en las 
bases de datos de WoS, analizando su evolución, así como sus patrones y tendencias.

2. Establecer cuáles son los autores y las instituciones con mayor producción de esta temática.

3. Identificar las posibles redes de coautoría y colaboración en los trabajos sobre narrativa EMA publicados en las bases de datos de WoS.

4. Determinar las redes de cocitación de los descriptores de los artículos de narrativa EMA.

\section{Metodología}

Este estudio es exploratorio descriptivo y longitudinal y en él se han utilizado fundamentalmente técnicas bibliométricas cuantitativas así como el análisis de redes sociales.

Podemos considerar esta investigación como de tipo inductivo, ya que tratamos de obtener conclusiones generales sobre la producción científica sobre narrativa en el campo de la educación matemática a partir de las publicaciones indexadas en las distintas bases de datos de la Web of Science.

Se han determinado indicadores de producción y de citación, tanto de autoría, como institucionales, por publicaciones y países, entre otras variables y, en lo relativo a la colaboración, se ha realizado un análisis de redes sociales (ARS) de autoría, colaboración institucional y entre los distintos países, además de analizarse la relación entre las temáticas a partir de los descriptores empleados por los autores en sus trabajos. Para ello se ha utilizado el programa Pajek (BATAGELJ; MRVRA, 2007).

La metodología empleada ha sido utilizada con anterioridad en estudios previos de características similares a éste, por lo que se puede considerar validada (EXPÓSITO; OLMEDO; FERNÁNDEZ-CANO, 2004; BRACHO, 2010; MAZ-MACHADO et al., 2012; BRACHO-LÓPEZ et al., 2012).

\subsection{Población y muestra}

Nos proponemos analizar la producción científica de relevancia a nivel internacional sobre narrativa EMA, pero dada la imposibilidad de acceder a toda la población, se ha hecho necesaria la elección de una muestra apropiada. Dada la aceptación de las bases de datos de Thomson Reuters como fuentes fundamentales de información y validación científicas, hemos 
optado por centrar nuestro estudio en los trabajos sobre narrativa EMA que aparecen en las publicaciones indexadas en las bases de datos de la Web of Science (FERNÁNDEZ-CANO, 2011).

\subsection{Búsqueda de documentos}

Las bases de datos accesibles a través de la Web of Science (WoS) permiten la realización de potentes búsquedas mediante la utilización de una versátil diversidad de campos relativos que, bien seleccionados y combinados mediante los operadores booleanos, nos pueden permitir buenos resultados en la localización de trabajos de la naturaleza que nos interesa. Básicamente, teníamos dos alternativas:

1. Usar el campo tópico e incorporar términos propios de la narrativa y de la educación matemática.

2. Ceñir la búsqueda a las cuatro revistas especializadas en educación matemática indexadas en la base SSCI y, si acaso, a las que no siendo específicas publican artículos de dicha área de conocimiento de manera más o menos sistemática, atendiendo en el mismo campo tópico a términos relacionados con la narrativa.

Sin duda, a falta de un deseado tesauro validado y aceptado internacionalmente que identificara eficazmente los trabajos sobre educación matemática, y dada la ambigüedad de este término al estar íntimamente relacionado con otras disciplinas como la psicología, la sociología, las matemáticas, la epistemología, la pedagogía e incluso la antropología (GUTIÉRREZ, 1991), la segunda opción hubiese sido la más sencilla, pero estaríamos desestimando a todos aquellos trabajos que son objeto de nuestro estudio pero no han sido publicados en revistas especializadas estrictamente en educación matemática. Por ello, nos hemos inclinado por la primera alternativa y, conscientes de la manifiesta ambigüedad de criterio a la hora de identificar a los trabajos de investigación en educación matemática que se evidencia en trabajos previos (BRACHO, 2010; LLINARES, 2008; MAZ; TORRALBO, 2007), hemos optado por utilizar la caracterización exhaustiva de descriptores de educación matemática que proponen Jiménez et al. (2011), y que precisamente fue validada en la utilización de la bases de datos de ciencias sociales de WoS y SCOPUS.

Para la determinación de los trabajos sobre narrativa entre los considerados de investigación en educación matemática, dentro del campo tópico se impusieron mediante el operador AND los criterios narrativ* or story* or biograph* or authobiog*. Posteriormente, 
se realizó un filtro en la base online por categorías para excluir revistas que no pertenezcan al campo de la educación.

Aplicando estos criterios de búsqueda y sin precisar el año de publicación, se obtuvieron inicialmente 379 trabajos (muestra disponible) entre los que, tras una primera consulta a expertos, se observó la inclusión en esta muestra inicial de numerosos trabajos que no estaban relacionados con la narrativa en educación matemática (ruido), por lo que se procedió a un filtrado manual basado fundamentalmente en la lectura de títulos, descriptores y resúmenes, obteniéndose un conjunto de 138 trabajos, que ya sí fueron validados por triangulación entre expertos como de narrativa EMA (muestra operante).

\section{Análisis de resultados}

\subsection{Producción diacrónica}

La distribución diacrónica de los 138 documentos hallados (Tabla 1) revela que esta producción científica es irregular y que se concentra en los últimos cinco años en los que se publica el 59,4\% del total. Puede verse como, prácticamente no aparecen trabajos sobre narrativa EMA hasta la década de los noventa del pasado siglo, siendo la presencia de éstos bastante discreta hasta 2006, cuando empieza a crecer más notablemente. Algo que parece lógico si tenemos en cuenta que la mayoría de los trabajos son artículos científicos (véase Tabla 4) y que el Journal for Research in Mathematic Education (JRME), la primera revista de educación matemática indexada en la base SSCI, lo es desde 1986, mientras que las otras tres revistas: Revista Latinoamericana de Investigación en Matemática Educativa (RELIME), Boletim de Educação Matematica (BOLEMA) y Educational Studies in Mathematics (ESM), son incluidas a partir de 2008 y 2009 respectivamente.

Tabla 1 - Producción diacrónica de documentos ISI-WoS sobre narrativa EMA

\begin{tabular}{cccccc}
\hline Año & $\begin{array}{c}\mathbf{N}^{\mathbf{0}} \mathbf{d e} \\
\text { publicaciones }\end{array}$ & $\mathbf{\%}$ & $\mathbf{A} \tilde{\mathbf{n o}}$ & $\begin{array}{c}\mathbf{N}^{\mathbf{0}} \text { de } \\
\text { publicaciones }\end{array}$ & $\%$ \\
\hline 1983 & 1 & 0,72 & 2003 & 4 & 2,90 \\
1992 & 2 & 1,45 & 2004 & 5 & 3,62 \\
1993 & 5 & 3,62 & 2005 & 3 & 2,17 \\
1995 & 3 & 2,17 & 2006 & 7 & 5,07 \\
1996 & 4 & 2,90 & 2007 & 8 & 5,80 \\
1997 & 2 & 1,45 & 2008 & 10 & 7,25 \\
1998 & 1 & 0,72 & 2009 & 17 & 12,32 \\
1999 & 5 & 3,62 & 2010 & 26 & 18,84 \\
\end{tabular}




\begin{tabular}{llllll}
2000 & 1 & 0,72 & 2011 & 14 & 10,14 \\
2001 & 4 & 2,90 & 2012 & 15 & 10,87 \\
2002 & 1 & 0,72 & & & \\
\hline
\end{tabular}

Fuente: desarrollado por los autores

\subsection{Autores}

Las publicaciones analizadas han sido firmadas por un total de 257 autores, entre los cuáles no existe ninguno que pueda considerarse gran productor de narrativa EMA, es decir, que publique más de 10 artículos sobre este tema. La gran mayoría $(93,39 \%)$ solo aportan una publicación, y tan solo existen 17 medianos productores (Tabla 2), por lo que se percibe una carencia aún de autores especializados en narrativa EMA, en el sentido de que presumiblemente centren sus investigaciones fundamentalmente en esta temática.

Tabla 2 - Autores con más trabajos publicados sobre narrativa EMA en ISI-WoS

\begin{tabular}{cccccc}
\hline Autor & $\mathbf{N}^{\mathbf{0}}$ trabajos & $\%$ & Autor & $\mathbf{N}^{\mathbf{0}}$ trabajos & $\%$ \\
\hline Kaasila, R & 4 & $2,90 \%$ & Mendick, H & 2 & $1,45 \%$ \\
Lutovac, S & 3 & $2,17 \%$ & Pajares, F & 2 & $1,45 \%$ \\
Aldridge, JM & 2 & $1,45 \%$ & Pampaka, M & 2 & $1,45 \%$ \\
Drake, C & 2 & $1,45 \%$ & Prawat, RS & 2 & $1,45 \%$ \\
Fraser, BJ & 2 & $1,45 \%$ & van den Heuvel, & 2 & $1,45 \%$ \\
Gadanidis, G & 2 & $1,45 \%$ & Wake, G & 2 & $1,45 \%$ \\
Herbst, P & 2 & $1,45 \%$ & Williams, K & 2 & $1,45 \%$ \\
Krysztofiak, W & 2 & $1,45 \%$ & Zeldin, AL & 2 & $1,45 \%$ \\
Meaney, T & 2 & $1,45 \%$ & & & \\
\hline
\end{tabular}

Fuente: desarrollado por los autores

Por otro lado, se observa un índice de colaboración $(\mathrm{IC}=2,007)$ similar al de dos firmas por trabajo, que Bordon y Gómez (1997) establecen para las ciencias sociales. Sin embargo, aproximadamente la mitad de los trabajos se publican de forma individual (50, 82\%). De esta forma, al representar la colaboración global en autoría se obtiene una red muy dispersa, con muchos nodos aislados y poca relación entre los autores, lo que denota una escasa colaboración sistemática. En la Figura 1 se representan las subredes más significativas en las que intervienen 5 autores o más. 


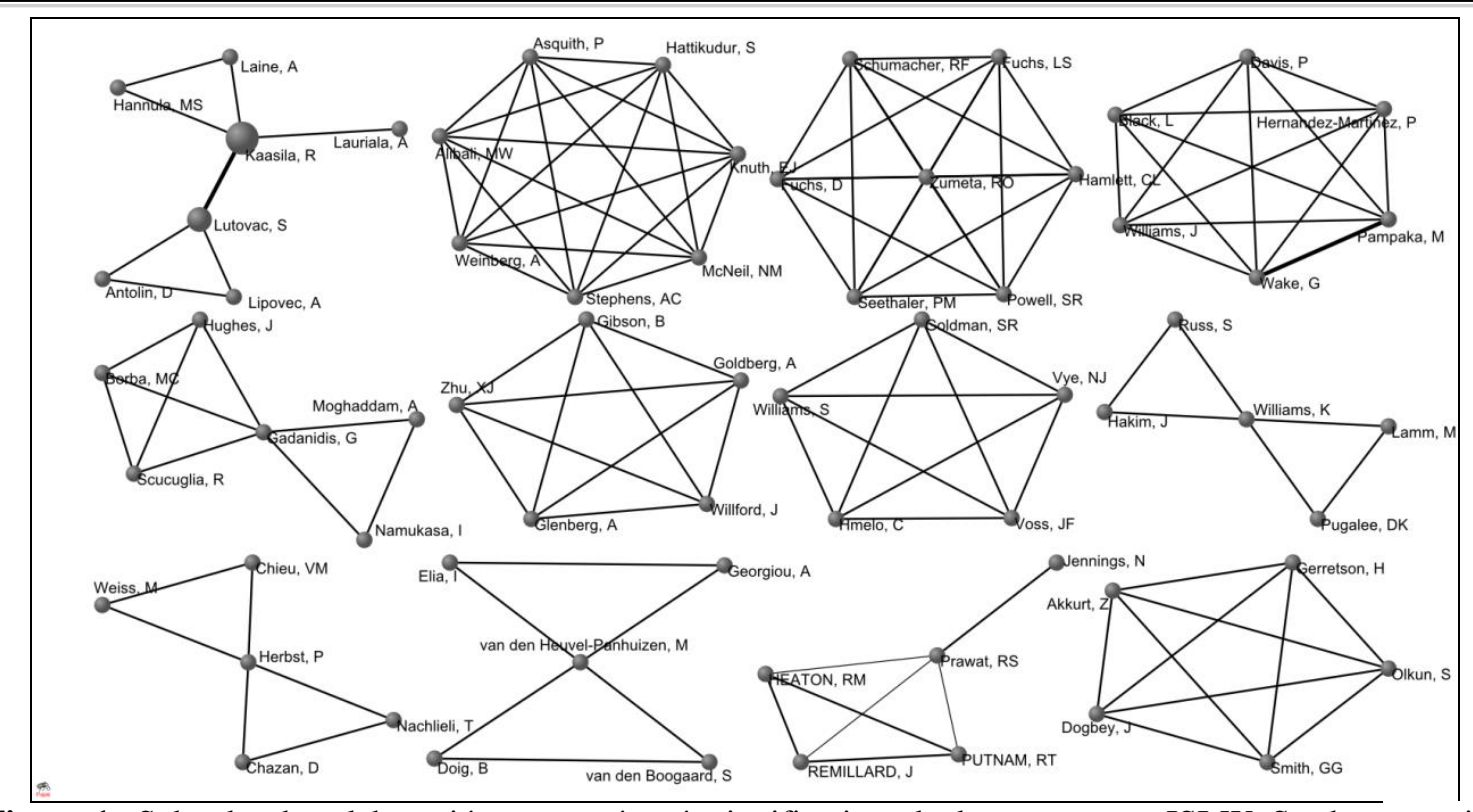

Figura 1- Subredes de colaboración en autoría más significativas de documentos en ISI-WoS sobre narrativa EMA

Fuente: desarrollado por los autores

\subsection{Citación de los trabajos}

El número total de citas recibidas por el conjunto de los 138 documentos que se analizan asciende a 822 . Gran parte de los trabajos (46,38\%) nunca han sido citados y este porcentaje sube hasta el 59,42\% si consideramos además a los que han recibido una sola cita, mientras que solo 11 trabajos acaparan más de la mitad de las citas recibidas (51,34\%).

Tabla 3 - Número de trabajos en ISI-WoS sobre narrativa EMA según su citación

$\begin{array}{cccc}\mathbf{N}^{\mathbf{0}} \text { citas } & \mathbf{N}^{\mathbf{0}} \text { trabajos } & \mathbf{N}^{\mathbf{0}} \text { citas } & \mathbf{N}^{\mathbf{0}} \text { trabajo } \\ 0 & 64 & 15 & 1 \\ 1 & 18 & 18 & 1 \\ 2 & 8 & 19 & 1 \\ 3 & 3 & 21 & 1 \\ 4 & 2 & 22 & 1 \\ 5 & 6 & 26 & 1 \\ 6 & 2 & 28 & 2 \\ 7 & 2 & 32 & 1 \\ 8 & 3 & 34 & 1 \\ 9 & 2 & 35 & 1 \\ 10 & 3 & 38 & 1 \\ 11 & 5 & 43 & 1 \\ 12 & 1 & 68 & 1 \\ 13 & 3 & 96 & 1 \\ 14 & 1 & & \end{array}$

Fuente: desarrollado por los autores 
Por trabajo, la media de citas que se obtiene es de 5,96, bastante por debajo de los índices establecidos en 2011 para la categoría Education \& Educational Research $(7,9)$ en el Journal Citation Reports® Social Sciences (THOMSON REUTER, 2013).

Los tres artículos más citados son Influences on classroom interest, de Bergin (1999 insertar en las referencias), publicado en Educational Psychologist, Against the odds: Selfefficacy beliefs of women in mathematical, scientific, and technological careers, de Zeldin y Pajares (2000 insertar en las referencias ), publicado en el American Educational Research Journal, y Anomalous information triggers questions when adults solve quantitative problems and comprehend stories, de Graesser y Mcmahen (1993 insertar en las referencias ), publicado en el Journal Of Educational Psychology, con 96, 68 y 43 citas, respectivamente. El primero es una investigación sobre los factores motivacionales que influyen en el aprendizaje del alumnado. El segundo trata de indagar en el autoconcepto relativo a la eficacia de mujeres que estudian carreras técnicas y cómo influye ésta en la superación de las mismas. El último artículo es una investigación sobre cómo la información errónea incide en la resolución de problemas y la comprensión de historias, resultando en un incremento de preguntas realizadas por los estudiantes.

El trabajo de Bergin (1999) podría considerarse clásico de citación, término acuñado por Garfield (1977) al referirse a los documentos altamente citados dentro de una determinada disciplina científica y que, en consecuencia, han podido influir notablemente en la investigación posterior dentro de la disciplina ${ }^{1}$.

\subsection{Tipología de los documentos. Revistas científicas donde aparecen los trabajos sobre narrativa EMA}

Según su tipología, la distribución de los documentos que se analizan es la siguiente:

Tabla 4 - Tipos de documentos en ISI-WoS sobre narrativa EMA

\begin{tabular}{ccc}
\hline Tipo de documento & $\mathbf{N}^{\mathbf{0}}$ de publicaciones & $\%$ \\
Revista & 114 & 82,61 \\
Serie, acta de congreso & 17 & 12,32 \\
Libro & 7 & 5,07 \\
\hline Fuente: desarrollado por los autores &
\end{tabular}

\footnotetext{
${ }^{1}$ Si bien Garfield (1977) no fijó un número de citas determinado para que un trabajo fuera considerado clásico de citación, hoy día se le suele aplicar ese distintivo a los trabajos que alcanzan o rondan las cien citas.
} 
Como era de esperar, las revistas científicas son el medio más utilizado para la difusión del conocimiento, seguidas de los trabajos presentados en congresos, o jornadas, de los que conviene comentar que la mayoría provienen de las sucesivas ediciones de la Conferencia Anual del Grupo Internacional de Psicología en Educación Matemática (PME) $(64,71 \%)$.

El abanico de las publicaciones en las que aparecen los 138 trabajos seleccionados se abre a 79 títulos, de los cuáles los que más se repiten corresponden a revistas científicas, entre las que destacan Teaching and Teacher Education, Educational Studies in Mathematics y Journal for Research in Mathematics Education con 8, 8 y 7 artículos respectivamente, siendo precisamente estas dos últimas publicaciones las de mayores factores de impacto entre las especializadas en EMA.

Tabla 5 - Revistas ISI-WoS más productivas en narrativa EMA (3 o más trabajos)

\begin{tabular}{lcc}
\hline \multicolumn{1}{c}{ Revista } & $\mathbf{N}^{\mathbf{0}}$ de artículos & $\mathbf{\%}$ \\
\hline Teaching and Teacher Education & 8 & 5,80 \\
Educational Studies in Mathematics & 8 & 5,80 \\
Journal for Research in Mathematics Education & 7 & 5,07 \\
Historia Mathematica & 5 & 3,62 \\
Gender and Education & 5 & 3,62 \\
American Educational Research Journal & 5 & 3,62 \\
Cognition and Instruction & 5 & 3,62 \\
Mathematics Education Bulletin (BOLEMA) & 4 & 2,90 \\
International Journal of Science and Mathematics Education & 3 & 2,17 \\
Journal of Curriculum Studies & 3 & 2,17 \\
Elementary School Journal & 3 & 2,17 \\
\hline Fuente: desarrodo por lositores
\end{tabular}

Fuente: desarrollado por los autores

\subsection{Nacionalidad y vinculación institucional de los autores}

Los 257 autores de los trabajos objeto de nuestro estudio pertenecen a 134 instituciones de 33 países diferentes. En la Tabla 6 se recogen los países que han contribuido con más de un trabajo. Es de destacar la esperada preponderancia de USA con 53 de las 138 aportaciones (38\%), al igual que la del idioma inglés en el que se escriben 130 de los trabajos analizados $(94,2 \%)$.

Tabla 6 - Países con 2 o más trabajos indexados en ISI-WoS sobre narrativa EMA

\begin{tabular}{lcclcc}
\hline \multicolumn{1}{c}{ País } & $\begin{array}{c}\mathbf{N}^{\mathbf{0}} \mathbf{d e} \\
\text { trabajos }\end{array}$ & $\mathbf{\%}$ & \multicolumn{1}{c}{ País } & $\begin{array}{c}\mathbf{N}^{\mathbf{0}} \text { de } \\
\text { trabajos }\end{array}$ & $\%$ \\
\hline USA & 53 & $38,41 \%$ & Chipre & 2 & $1,45 \%$ \\
Canadá & 8 & $5,80 \%$ & Francia & 2 & $1,45 \%$ \\
Inglaterra & 8 & $5,80 \%$ & Japón & 2 & $1,45 \%$ \\
Australia & 7 & $5,07 \%$ & Malasia & 2 & $1,45 \%$
\end{tabular}




\begin{tabular}{llllll}
\hline Finlandia & 6 & $4,35 \%$ & Norruega & 2 & $1,45 \%$ \\
Alemania & 4 & $2,90 \%$ & Polonia & 2 & $1,45 \%$ \\
Holanda & 4 & $2,90 \%$ & Singapur & 2 & $1,45 \%$ \\
Nueva Zelanda & 4 & $2,90 \%$ & España & 2 & $1,45 \%$ \\
Brasil & 3 & $2,17 \%$ & Suecia & 2 & $1,45 \%$ \\
Israel & 3 & $2,17 \%$ & Suiza & 2 & $1,45 \%$ \\
Eslovenia & 3 & $2,17 \%$ & Taiwan & 2 & $1,45 \%$ \\
Sudáfrica & 3 & $2,17 \%$ & & & \\
\hline
\end{tabular}

Fuente: desarrollado por los autores

En la Figura 2 se representa la colaboración entre autores de diferentes países. En ella, el tamaño del nodo indica el volumen de la producción, mientras que el grosor de las líneas nos da una idea del grado de colaboración entre los países que se relacionan. Como puede observarse, son sólo 13 los países que han publicado en colaboración y la relación entre estos países también es escasa. Tan solo puede hablarse de cierto nivel de colaboración entre Países Bajos, Australia y Alemania.

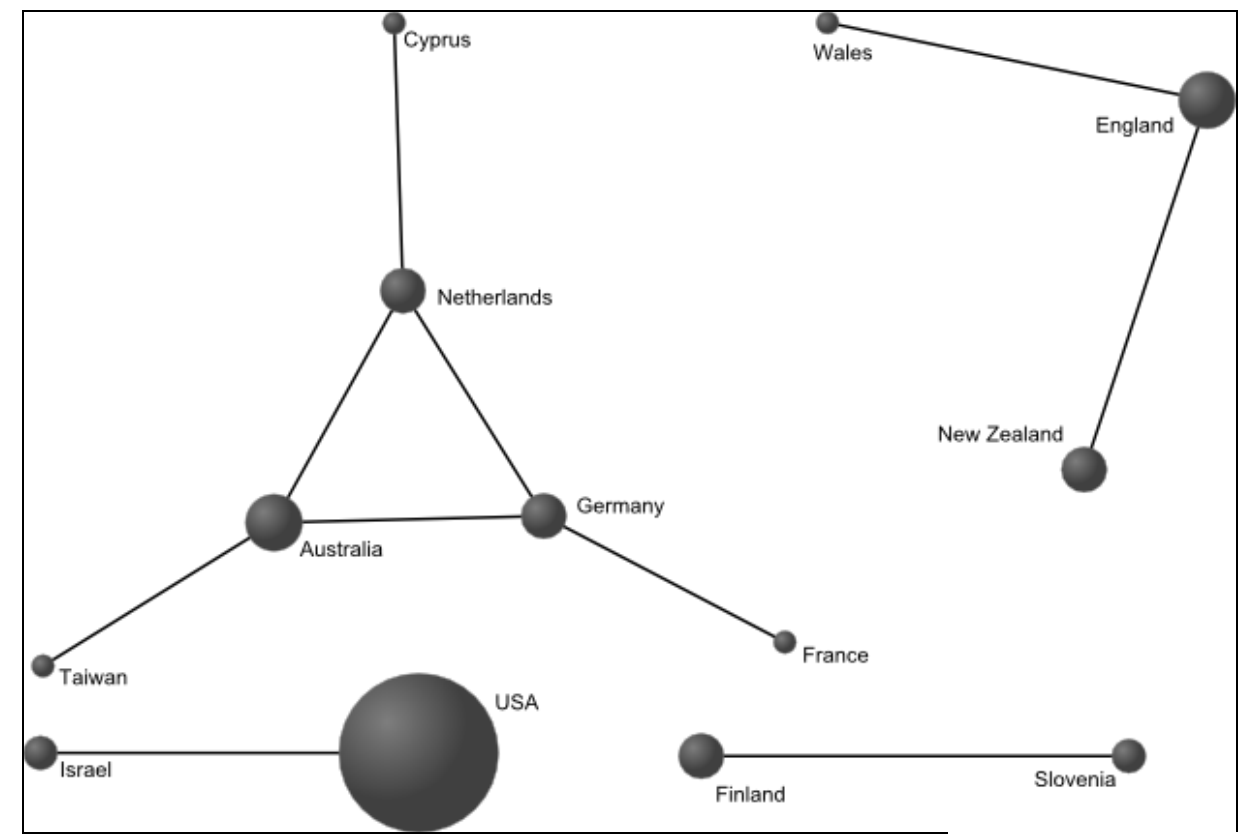

Figura 2 - Red de colaboración entre países productores de documentos sobre narrativa EMA indexados en ISIWoS

Fuente: desarrollado por los autores

Respecto a las instituciones, 115 de las 134, es decir, el 85,22 \%, son universidades. Por otro lado, la gran mayoría de las instituciones publican un solo trabajo sobre narrativa EMA (84,06 \%). Las que publican más de un trabajo están recogidas en la tabla siguiente: 
Tabla 7 - Ranking de instituciones más productivas (más de una publicación) en documentos sobre narrativa EMA indexados en ISI-WoS

\begin{tabular}{|c|c|c|c|c|c|}
\hline Institución & $\mathrm{N}^{\circ}$ trabajos & $\%$ & Institución & $\mathrm{N}^{\circ}$ trabajos & $\%$ \\
\hline Univ Michigan & 5 & $3,62 \%$ & Univ Auckland & 2 & $1,45 \%$ \\
\hline Univ Lapland & 4 & $2,90 \%$ & $\begin{array}{l}\text { Univ British } \\
\text { Columbia }\end{array}$ & 2 & $1,45 \%$ \\
\hline $\begin{array}{l}\text { Michigan State } \\
\text { Univ }\end{array}$ & 3 & $2,17 \%$ & Univ Geneva & 2 & $1,45 \%$ \\
\hline Univ Utrecht & 3 & $2,17 \%$ & Univ Manchester & 2 & $1,45 \%$ \\
\hline Adelphi Univ & 2 & $1,45 \%$ & Univ Maribor & 2 & $1,45 \%$ \\
\hline $\begin{array}{c}\text { Charles Sturt } \\
\text { Univ }\end{array}$ & 2 & $1,45 \%$ & Univ Pittsburgh & 2 & $1,45 \%$ \\
\hline Emory Univ & 2 & $1,45 \%$ & UnivPutra & 2 & $1,45 \%$ \\
\hline $\begin{array}{c}\text { Georgia State } \\
\text { Univ }\end{array}$ & 2 & $1,45 \%$ & Univ Wisconsin & 2 & $1,45 \%$ \\
\hline $\begin{array}{c}\text { Simon } \\
\text { FraserUniv }\end{array}$ & 2 & $1,45 \%$ & Vanderbilt Univ & 2 & $1,45 \%$ \\
\hline
\end{tabular}

Fuente: desarrollado por los autores

En cuanto a la colaboración institucional, también se observa poca relación entre las universidades más productivas, como puede observarse en la red de colaboración institucional (Figura 3). Esto indica que la colaboración en la autoría es mayoritariamente de tipo interinstitucional y, por lo tanto, local.

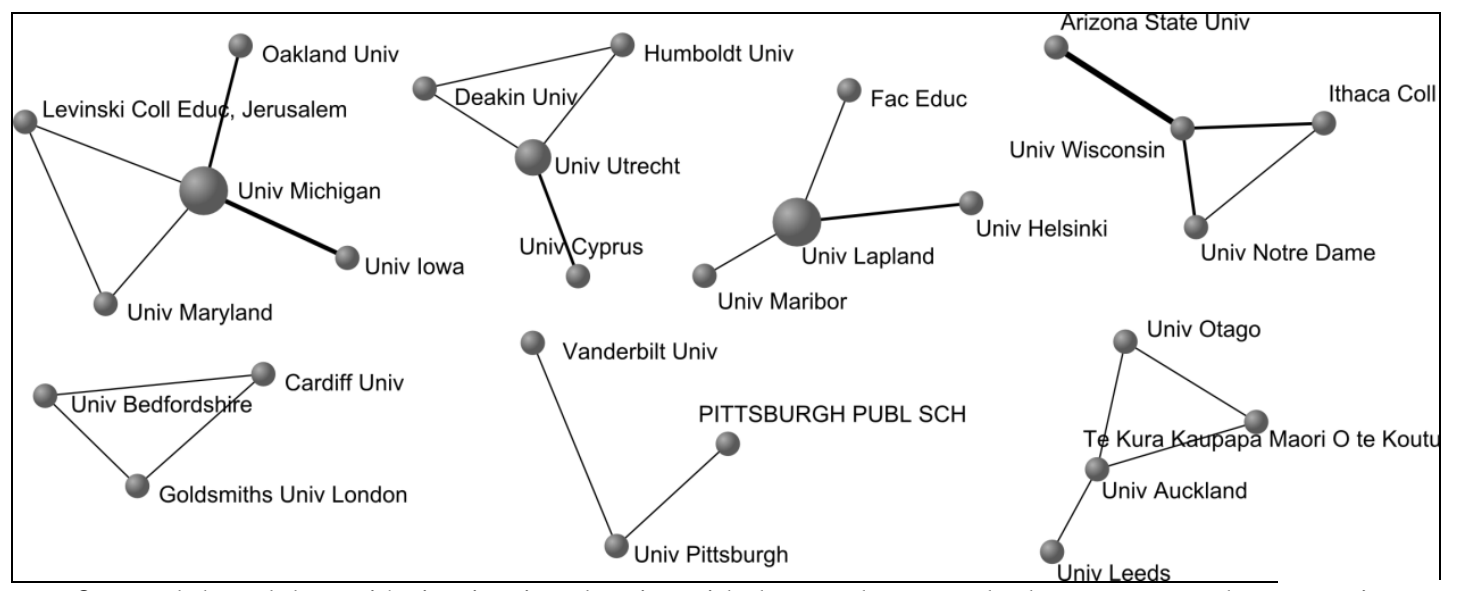

Figura 3 - Red de colaboración institucional-universidades productoras de documentos sobre narrativa EMA indexados en ISI-WoS

Fuente: desarrollado por los autores

\subsection{Relación entre los descriptores}

65 de los 138 trabajos que se han analizado no han sido categorizados por sus autores, por lo que este estudio de la relación entre los descriptores se ha centrado en el 52,9\% restante. Estas publicaciones generan un total de 275 descriptores que, tras una pequeña normalización, se quedan en 273.

La red de descriptores se muestra a continuación en la Figura 4. 


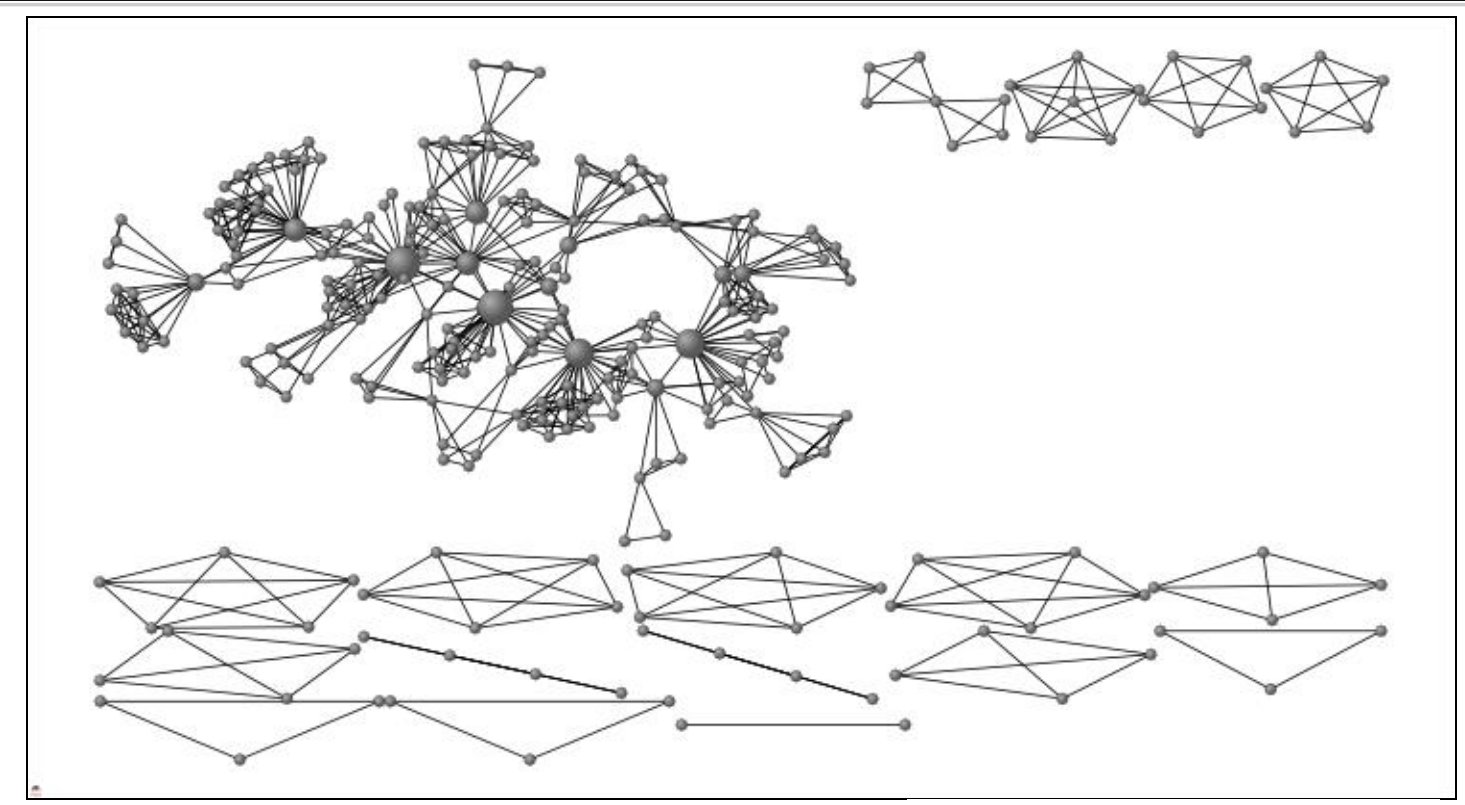

Figura 4 - Red de descriptores (global) en documentos sobre narrativa EMA indexados en ISI-WoS Fuente: desarrollado por los autores

Como se observa, existe una gran red que aglutina la mayor parte de descriptores específicos de narrativa utilizados en la búsqueda. El tamaño de los nodos representa la frecuencia con la que se utiliza cada descriptor. No se presentan las subredes integradas exclusivamente por un documento al interpretarse que los términos en cuestión no son ampliamente utilizados.

Centrándonos en la gran subred y dada la complejidad de la misma, la analizaremos obteniendo a partir de esta las subredes de los descriptores principales, a saber: narrative, biography y story. Para ellos buscaremos estas palabras-clave en los campos descriptores, título y resumen de la muestra operante.

En la subred asociada al término narrative (Figura 5) se observa que este descriptor está fuertemente vinculado con los descriptores relativos a la formación de profesores (incluyendo profesores de matemáticas), desarrollo profesional, actitudes, autoeficacia, identidad etc., todos ellos aspectos notables del perfil profesional del profesor de matemáticas. 


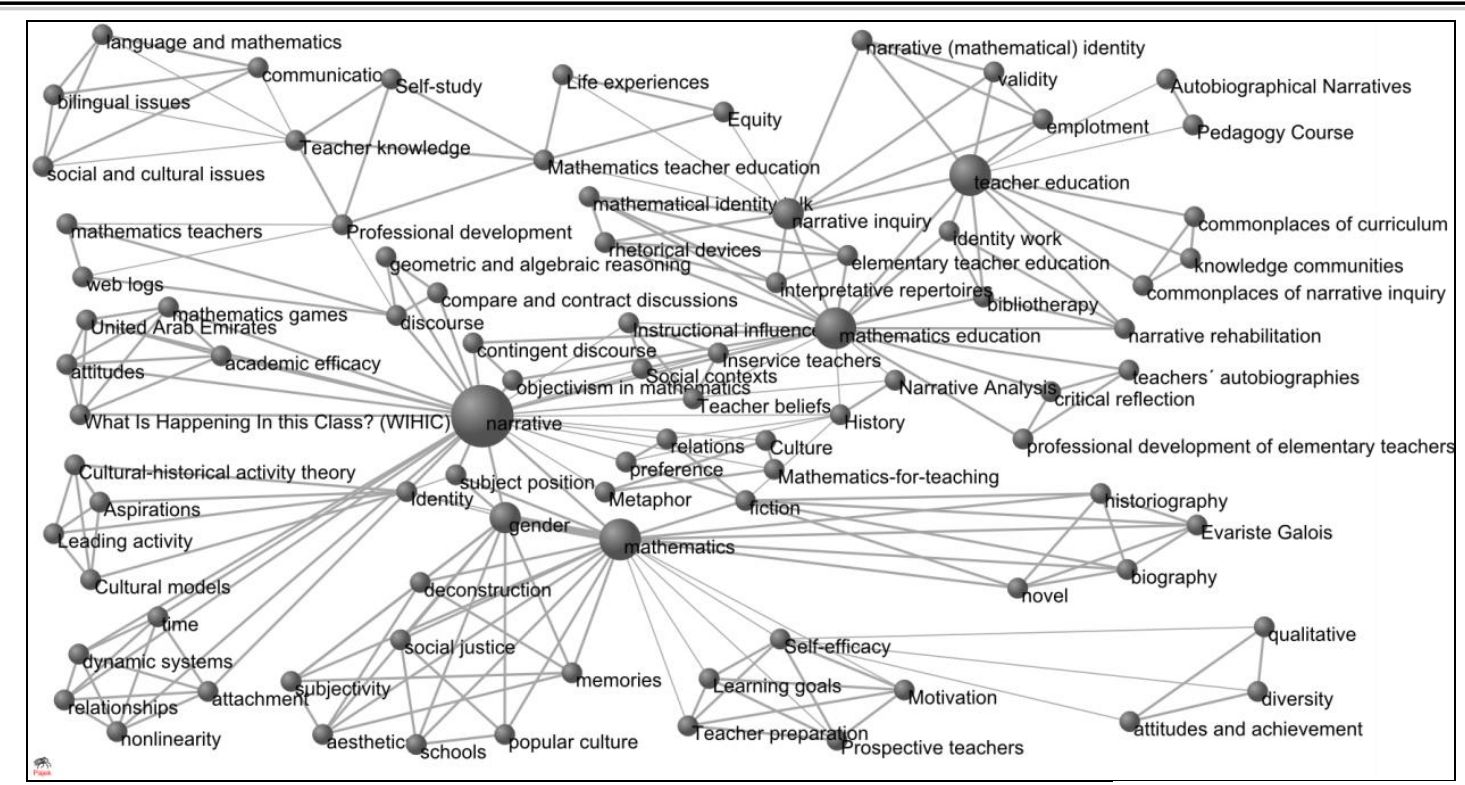

Figura 5 - Subred de cocitación de descriptores (narrative) en documentos sobre narrativa EMA indexados en ISI-WoS

Fuente: desarrollado por los autores

Observando la subred asociada al término biography podemos concluir que este descriptor está ligado principalmente con la educación matemática y, a través de esta, con la formación de profesores, el desarrollo profesional y alumnado. Se observa una conexión con la rama de la historia de las matemáticas y la historia de la educación matemática.

Para el término story se obtienen 21 subredes. Así, se puede observar cómo la palabraclave story está relacionada con muchas más temáticas, existiendo una fuerte relación con términos propios de la formación de profesores, como en las redes halladas para las palabrasclave que ya hemos comentado. Pero observamos también, el uso de historias en la resolución de problemas y las historias como narrativas del alumnado, sobretodo en las primeras etapas.

\section{Conclusiones}

Si bien la educación matemática es una disciplina consolidada como tal a nivel internacional desde hace años, no cabe duda de que se trata de un área de conocimiento respaldada por una comunidad científica todavía exigua en términos comparativos, aunque creciente, como demuestra la modesta y reciente nómina de publicaciones especializadas indexadas en la base de datos SSCI. En este trabajo se ha podido constatar cómo la producción en narrativa EMA indexada en las bases de datos de la WoS dentro de este área de conocimiento, aunque moderada en conjunto a lo largo del tiempo, ha ido emergiendo hasta alcanzar cierto nivel de producción y regularidad en los últimos años, en consonancia con el 
papel importante que la narrativa ha ido ocupando en la investigación educativa en general.

Como consecuencia de esta corta historia productiva, no puede hablarse aún de la existencia de grandes autores en esta temática en las bases de WoS, si bien aunque lógicamente escasos todavía, ya existen algunos referentes que acaparan el interés de especialistas.

En el plano institucional ya se vislumbra la clara preponderancia de las universidades anglosajonas, en especial estadunidenses, aunque quizá la incorporación, todavía reciente, a la base SSCI de dos revistas latinoamericanas específicas de educación matemática: una mexicana y otra brasileña, podría frenar este posible sesgo a medio o largo plazo e incrementar también la presencia de trabajos en español y portugués. Por otro lado, el nivel de colaboración institucional y entre países es muy escaso todavía, observándose también que nos encontramos ante una temática en fase de despegue en la que los colegios invisibles en el plano internacional se encuentran todavía por configurar.

Respecto a las temáticas, las redes de descriptores señalan que la producción de artículos relacionados con la narrativa en EMA tiene unos vínculos orientados hacia la resolución de problemas, la formación del profesorado y la historia de la matemática y de la propia disciplina.

Dentro de las limitaciones de este estudio, se encuentra la propia naturaleza del WoS en cuanto al número y tipos de revistas que indexa en su cobertura geográfica, así como el hecho de que al no existir un tesauro específico de EMA, los autores vayan creando descriptores ad hoc, generándose así una serie de descriptores definidos de forma subjetiva y dejando a criterio del autor la determinación de si el término es adecuado o no. Como afirman algunos autores "esta libertad de descriptores afecta no solo a la visibilidad de los autores, sino que también sus aportes en su campo de investigación se diluyen en el ingente volumen de información de la investigación educativa" (ADAMUZ-POVEDANO; JIMÉNEZFANJUL; MAZ-MACHADO, 2013, p. 2).

Resumiendo, se constata que nos encontramos ante una temática aún en ciernes pero con indicios de convertirse en emergente en los próximos años, que podría complementar significativamente la investigación en educación matemática con la dimensión histórica, social y cultural que se demanda desde la sociedad en general, y desde el mundo de la educación en particular.

En este sentido, pensamos que podría resultar interesante el planteamiento de una réplica de este trabajo en el trascurso de unos años para verificar el estado de la cuestión en el 
que también se podría abordar la información de otras bases que otorguen mayor cobertura a revistas de educación en general y de EMA, en particular de habla no inglesa, como pueden ser SCOPUS o MathEdu.

\section{Referencias}

ADAMUZ-POVEDANO, N.; JIMÉNEZ-FANJUL, N.; MAZ-MACHADO, A. Búsqueda de descriptores que caractericen una disciplina emergente en WoS y SCOPUS: el caso de la Educación Matemática. Biblios - Revista Electrónica de Bibliotecología, Archivología y Museología, Lima, n. 50, p. 1-14, abr. 2013

BATAGELJ, V.; MRVAR, A. Pajek software Descargado el 12 de febrero de 2009. 2007. Disponible en: 〈http://pajek.imfm.si/doku.php〉. Consultado en: 21 jul. 2014.

BEHRENS, H.; LUKSCH, P. Mathematics 1868-2008: a bibliometric analysis. Scientometrics, Budapest, v. 86, n. 1, p. 179-194, ene. 2011.

BERGIN. D. A. Influences on classroom interest. Educational Psychologist, Hillsdale, v. 34, n. 2, p. 87-98, jun. 1999.

BORDON, M.; GÓMEZ, I. La actividad científica española a través de los indicadores bibliométricos en el periodo 1990-93. Revista General de Información y Documentación, Madrid, v.7, n. 2, p. 69 86, dic. 1997.

BRACHO, R. Visibilidad de la investigación en educación matemática en España. Análisis cienciométrico y conceptual de la producción de artículos científicos. 2010. 123-229 f. Tesis (Doctoral en Educación Matemática) - Facultad de Educación, Universidad de Córdoba, Córdoba, 2010.

BRACHO-LÓPEZ, R.; MAZ-MACHADO, A.; GUTIÉRREZ-ARENAS, P.; TORRALBORODRÍGUEZ, M.; JIMÉNEZ-FANJUL, N.; ADAMUZ-POVEDANO, N. La investigación en educación matemática a través de las publicaciones científicas españolas. Revista Española de Documentación Científica, Madrid, v. 35, n. 2, p. 262-280, jun. 2012.

CURY, F. G. Análise narrativa emtrabalhos de história da educação matemática: algumas considerações. Bolema: Mathematics Education Bulletin, Rio Claro, v. 23, n. 35, p. 59-73, abr. 2010.

DONOGHUE, E. F. The task-teqnique matrix: analternative system for classifying research in mathematics education. School Science and Mathematics, Stillwater, OK, v. 99, n. 1, p. 42-46, ene. 1999.

ERNEST, P. A. Postmodern perspective on research in mathematics education. In: SIERPINSKA, A.; KILPATRICK, J. (Coord.). Mathematics education as a research domain: a search for identity. An ICMI study. Dordrech: Kluwer Academic Publishers, 1998. p. 71-86.

EXPÓSITO, J; OLMEDO, E.; FERNÁNDEZ-CANO, A. Patrones metodológicos en la investigación española sobre evaluación de programas educativos. RELIEVE, Valencia, v. 10, n. 2, p. 185-209, jul. 2004.

FERNÁNDEZ-CANO, A. Producción educativa española en el Social Sciences Citation Index (19982009). II. Revista Española de Pedagogía, Madrid, v. 69, n. 250, p. 427-443, dic. 2011. 
GARCÍA-MARTÍNEZ, A. T.; GUERRERO-BOTE, V.; MOYA-ANEGÓN, F. World scientific production in Psychology. Universitas Psychologica, Bogotá, v. 11, n. 3, p. 699-717, 2012.

GARFIELD, E. Introducing Citation Classics: The human side of scientific papers. Current Contents, Filadelfia, v. 1, n. 3, p. 1-2, ene. 1977.

GODINO, J. D. Hacia una teoría de la didáctica de la matemática. En: GUTIÉRREZ, A. (Ed.). Área de conocimiento didáctica de la matemática. Madrid: Síntesis, 1991. p. 105-148.

GODINO, J. D., CARRILLO, J.; CASTRO, W.; LACASTA, E.; MUÑOZ-CATALÁN, M. C.; WILHELMI, M. R. Métodos de investigación en las ponencias y comunicaciones presentadas en los simposios de la SEIEM. Avances de Investigación en Educación Matemática, Badajoz, n. 2, p. 2952, mayo 2012.

GRAESSER, A. C.; MCMAHEN, C. L. Anomalous information triggers questions when adults solve quantitative problems and comprehend stories. Journal of Educational Psychology, Menphis, v. 85, n. 1, p. 136-151, ma. 1993.

GUTIÉRREZ, A. La investigación en Didáctica de las Matemáticas. En: GUTIÉRREZ, A. (Ed.). Área de conocimiento: Didáctica de las Matemáticas. Madrid: Síntesis, 1991. p. 149-194.

JIMÉNEZ, N.; ADAMUZ, N.; MAZ, A.; BRACHO, R.; LUPIÁÑEZ, J. L.; SEGOVIA, I. Producción científica internacional en Educación Matemática en SSCI y SCOPUS (1980-2009): Construcción de descriptores. En: LUPIÁÑEZ J. L. et al. (Coord.). Pensamiento numérico y algebraico e historia de las matemáticas y educación matemática. Granada: Universidad de Granada, 2011. p. 325-335.

JOHNSON, D. C. Types of research. En: SHUMWAY, R. J. (Ed.). Research in mathematics education. Reston, VA: NCTM,1980a. p. 286-323.

JOHNSON, D. C. The research process. En: SHUMWAY, R. J. (Ed.). Research in mathematics education. Reston, VA: NCTM, 1980b. p. 29-46.

LLINARES, S. Agendas de Investigación en Educación Matemática en España. Una aproximación desde "ISI-web of knowledge" y ERIH. En: LUENGO, G. et al. (Coord.). Investigación en Educación Matemática XII. Badajoz: Sociedad Extremeña de Educación Matemática "Ventura Prosper"-SEIEM, 2008. p. 25-53.

MAZ, A.; TORRALBO, M. Producción ISI del profesorado universitario español del área de Didáctica de la Matemática. En: CAMACHO, M. et al. (Coord.). Investigación en Educación Matemática. Comunicaciones de los grupos de investigación. XI Simposio de la SEIEM. Tenerife: SEIEM, 2007. p. 181-188.

MAZ-MACHADO, A. et al. Estudio bibliométrico de la investigación educativa en las universidades de Andalucía en el SSCI (2002-2010). Revista Iberoamericana de Psicología y Salud, A Coruña, v. 3, n. 2, p. 125-136, 2012.

MISKULIN, R. G. S.; PENTEADO, M. G.; RICHIT, A.; MARIANO, C. R. A Prática do professor que ensina matemática e a colaboração: uma reflexão a partir de processos formativos virtuais. Bolema: Mathematics Education Bulletin, Rio Claro, v. 25, n. 41, p. 173-186, 2012.

SIERPINSKA, A.; KILPATRICK, J. Continuing the search. En: SIERPINSKA, A.; KILPATRICK, J. (Coord.). Mathematics education as a research domain: a search for identity. An ICMI study. Dordrech: Kluwer Academic Publishers, 1998. p. 527-548. 
STEINER, H. G. Needed cooperation between science education and mathematics education. ZDMThe International Journal on Mathematics Education, Dordrecht, v. 60, n. 6, p. 194-197, 1990.

THOMSON REUTERS, 2013. Disponible en: <http://thomsonreuters.com/journal-citation-reports/>. Consultado en: 21 jul. 2014.

TORRALBO, M.; VALLEJO, M.; FERNÁNDEZ-CANO, A.; RICO, L. Análisis metodológico de la producción española de tesis doctorales en educación matemática (1976-1998). Revista Electrónica de Investigación y Evaluación Educativa, Valencia, v. 10, n. 1, 41-59, ene. 2004.

ZELDIN, A. L.; PAJARES, F. Against The Odds: Self-Efficacy Beliefs Of Women In Mathematical, Scientific, And Technological Careers. American Educational Research Journal, California, v. 37, n. 1, p. 215-246, mar. 2000.

Submetido em Agosto de 2013. Aprovado em Janeiro de 2014. 